RAFAŁ KuliK (Wrocław)

\title{
STOCHASTIC COMPARISON OF MULTIVARIATE RANDOM SUMS
}

Abstract. We establish preservation results for the stochastic comparison of multivariate random sums of stationary, not necessarily independent, sequences of nonnegative random variables. We consider convex-type orderings, i.e. convex, coordinatewise convex, upper orthant convex and directionally convex orderings. Our theorems generalize the well-known results for the stochastic ordering of random sums of independent random variables.

1. Introduction. In this paper we establish preservation results for the stochastic comparison of multivariate random sums of nonnegative random variables. We consider convex, coordinatewise convex, upper orthant convex and directionally convex orderings. The first three are variability orderings, whereas the latter can be considered as a variability-dependence ordering. We refer the reader to the books [7] or [10]. Using so called supermodular functions we are able to extend the results known in the case of independent random variables ([2], [8], [9]) to stationary sequences.

Random sums are used in many applied sciences, and comparison of such sums plays an important role in stochastic models, where exact calculations of some quantities are not possible. For example in the context of multivariate shock models preservation of the stochastic ordering for (multivariate) random sums was established in [8] and [12]. In actuarial science random sums were considered in [1], and in the context of queueing systems in [2] and [4]. In the latter paper a preservation result was proved (in the directionally convex case) not only for random sums but for more general functionals of stationary point processes.

The paper is organized as follows. In Section 2 we collect some needed definitions and technical lemmas. In Section 3 we prove regularity properties

2000 Mathematics Subject Classification: Primary 60E15.

Key words and phrases: multivariate random sums, multivariate stochastic orders, convex order, directionally convex order, supermodular function. 
of sums of random variables and then we apply them to comparison results. In Section 4 we point out some cases when the assumptions of the main theorems are satisfied, i.e. we present examples of vectors of integer-valued random variables which can be compared in the above mentioned stochastic orderings.

2. Preliminaries. We shall consider stationary sequences of nonnegative random variables. Precisely, we say that sequences $\left\{U_{n}^{i}\right\}_{n \geq 1}, i=1, \ldots, k$, are jointly stationary if for all $n_{i} \geq 1, i=1, \ldots, k, m \geq 1$,

$$
\begin{aligned}
\left(\left(U_{1}^{1}, \ldots, U_{n_{1}}^{1}\right), \ldots,\right. & \left.\left(U_{1}^{k}, \ldots, U_{n_{k}}^{k}\right)\right) \\
& \stackrel{\mathrm{d}}{=}\left(\left(U_{1+m}^{1}, \ldots, U_{n_{1}+m}^{1}\right), \ldots,\left(U_{1+m}^{k}, \ldots, U_{n_{k}+m}^{k}\right)\right),
\end{aligned}
$$

where $\stackrel{\mathrm{d}}{=}$ denotes equality in distribution.

We shall use some special classes of functions. A function $\varphi: \mathbb{R}^{k} \rightarrow \mathbb{R}$ is convex on $\mathbb{R}^{k}$ if for all $\mathbf{u}=\left(u_{1}, \ldots, u_{k}\right), \mathbf{v}=\left(v_{1}, \ldots, v_{k}\right)$ and $\alpha \in(0,1)$ we have $\varphi(\alpha \mathbf{u}+(1-\alpha) \mathbf{v}) \leq \alpha \varphi(\mathbf{u})+(1-\alpha) \varphi(\mathbf{v})$. For the convexity on $\mathbb{N}$ $(k=1)$ this condition can be written as $\varphi(n+1)+\varphi(n-1) \geq 2 \varphi(n)$ for all $n \geq 1$.

For $1 \leq i \leq k, \varepsilon>0$ and an arbitrary function $\varphi: \mathbb{R}^{k} \rightarrow \mathbb{R}$ define the difference operator $\Delta_{i}^{\varepsilon}$ by

$$
\Delta_{i}^{\varepsilon} \varphi\left(u_{1}, \ldots, u_{k}\right)=\varphi\left(u_{1}, \ldots, u_{i-1}, u_{i}+\varepsilon, u_{i+1}, \ldots, u_{k}\right)-\varphi\left(u_{1}, \ldots, u_{k}\right)
$$

for given $u_{1}, \ldots, u_{k}$. A function $\varphi: \mathbb{R}^{k} \rightarrow \mathbb{R}$ is called

(i) coordinatewise convex if for all $1 \leq i \leq k$ and $\varepsilon_{i}, \varepsilon_{j}>0$,

$$
\Delta_{i}^{\varepsilon_{i}} \Delta_{i}^{\varepsilon_{j}} \varphi(\mathbf{u}) \geq 0 \quad \text { for all } \mathbf{u}=\left(u_{1}, \ldots, u_{k}\right)
$$

(ii) supermodular if for all $1 \leq i<j \leq k$ and $\varepsilon_{i}, \varepsilon_{j}>0$,

$$
\Delta_{i}^{\varepsilon_{i}} \Delta_{j}^{\varepsilon_{j}} \varphi(\mathbf{u}) \geq 0 \quad \text { for all } \mathbf{u}=\left(u_{1}, \ldots, u_{k}\right)
$$

(iii) directionally convex if it is supermodular and coordinatewise convex, or equivalently, for all $1 \leq i \leq j \leq k$ and $\varepsilon_{i}, \varepsilon_{j}>0$,

$$
\Delta_{i}^{\varepsilon_{i}} \Delta_{j}^{\varepsilon_{j}} \varphi(\mathbf{u}) \geq 0 \quad \text { for all } \mathbf{u}=\left(u_{1}, \ldots, u_{k}\right) .
$$

We shall say that the function is coordinatewise convex (resp. supermodular, directionally convex) on $\mathbb{N}^{k}$ if the above conditions hold for $\mathbf{u}=$ $\left(u_{1}, \ldots, u_{k}\right) \in \mathbb{N}^{k}$ and $\varepsilon_{i}, \varepsilon_{j}=1$.

Moreover, we say a function $\varphi: \mathbb{R}^{k} \rightarrow \mathbb{R}$ is of upper orthant (resp. lower orthant, convex upper orthant) type if it has the form

$$
\varphi\left(u_{1}, \ldots, u_{k}\right)=\prod_{i=1}^{k} h_{i}\left(u_{i}\right),
$$

where $h_{i}: \mathbb{R} \rightarrow \mathbb{R}$ are increasing (resp. decreasing, increasing and convex). 
We denote by $\mathcal{L}_{\text {cx }}\left(\right.$ resp. $\mathcal{L}_{\text {icx }}, \mathcal{L}_{\text {ccx }}, \mathcal{L}_{\text {iccx }}, \mathcal{L}_{\text {sm }}, \mathcal{L}_{\text {dcx }}, \mathcal{L}_{\text {idcx }}, \mathcal{L}_{\text {uo }}, \mathcal{L}_{\text {lo }}$, $\mathcal{L}_{\text {uo-cx }}$ ) the class of convex (resp. increasing and convex, coordinatewise convex, increasing and coordinatewise convex, supermodular, directionally convex, increasing and directionally convex, upper orthant type, lower orthant type, convex upper orthant type) functions. Here, "increasing" or "decreasing" is meant in the weak sense.

Recall that $\mathcal{L}_{\text {idcx }} \subset \mathcal{L}_{\text {dcx }} \subset \mathcal{L}_{\text {sm }}$ and $\mathcal{L}_{\text {dcx }} \subset \mathcal{L}_{\text {ccx }}$. Note that a function which is directionally convex need not be convex, and a function which is convex need not be directionally convex. Consider for example $\varphi\left(u_{1}, \ldots, u_{k}\right)$ $=\max \left(u_{1}, \ldots, u_{k}\right)$, which is convex, but not directionally convex.

EXAMPLE 2.1. We present some examples of functions which are members of the above classes. Let $u_{i} \geq 0, i=1, \ldots, k$.

1. $\varphi\left(u_{1}, \ldots, u_{k}\right)=f\left(\sum_{i=1}^{k} u_{i}\right) \in \mathcal{L}_{\mathrm{dcx}}$ for $f: \mathbb{R} \rightarrow \mathbb{R}_{+}$convex;

2. $\varphi\left(u_{1}, \ldots, u_{k}\right)=f\left(\prod_{i=1}^{k} u_{i}\right) \in \mathcal{L}_{\mathrm{dcx}}$ for $f: \mathbb{R} \rightarrow \mathbb{R}_{+}$increasing and convex;

3. $\varphi\left(u_{1}, \ldots, u_{k}\right)=\sum_{i=1}^{k} f\left(u_{i}\right) \in \mathcal{L}_{\mathrm{dcx}}$ for $f: \mathbb{R} \rightarrow \mathbb{R}_{+}$convex;

4. $\varphi\left(u_{1}, \ldots, u_{k}\right)=\prod_{i=1}^{k} f\left(u_{i}\right) \in \mathcal{L}_{\mathrm{dcx}}$ for $f: \mathbb{R} \rightarrow \mathbb{R}_{+}$increasing convex.

The above classes of functions generate stochastic orderings. For random vectors $\mathbf{U}=\left(U_{1}, \ldots, U_{k}\right), \widetilde{\mathbf{U}}=\left(\widetilde{U}_{1}, \ldots, \widetilde{U}_{k}\right)$ we write

$$
\mathbf{U}<_{\mathrm{a}} \widetilde{\mathbf{U}}
$$

if $\mathbb{E}[f(\mathbf{U})] \leq \mathbb{E}[f(\widetilde{\mathbf{U}})]$ for all $f \in \mathcal{L}_{\mathrm{a}}$ for which the expectations exist.

We shall need the following technical lemma.

Lemma 2.2. Let $\mathbf{u}=\left(u_{1}, \ldots, u_{k}\right)$.

(i) Let $f: \mathbb{R} \rightarrow \mathbb{R} \in \mathcal{L}_{\mathrm{cx}}$. Then $\phi: \mathbb{R}^{k} \rightarrow \mathbb{R}$ defined as

$$
\phi(\mathbf{u})=f\left(\sum_{i=1}^{k} u_{i}\right)
$$

is supermodular (and directionally convex) on $\mathbb{R}_{+}^{k}$.

(ii) Let $\varphi: \mathbb{R}^{k} \rightarrow \mathbb{R} \in \mathcal{L}_{\mathrm{dcx}}$ and $u_{i}^{j} \geq 0,1 \leq j \leq k, 1 \leq i \leq n_{j}$. Then $\phi$ defined as

$$
\phi\left(\left(u_{1}^{1}, \ldots, u_{n_{1}}^{1}\right), \ldots,\left(u_{1}^{k}, \ldots, u_{n_{k}}^{k}\right)\right)=\varphi\left(\sum_{i=1}^{n_{1}} u_{i}^{1}, \ldots, \sum_{i=1}^{n_{k}} u_{i}^{k}\right)
$$

is directionally convex on $\mathbb{R}_{+}^{n_{1}+\ldots+n_{k}}$.

Proof. (i) is taken from [6, p. 152]. In order to obtain (ii) we proceed as follows. 
Let $n=n_{1}+\ldots+n_{k}$. We need to show that $\Delta_{i}^{\varepsilon_{i}} \Delta_{j}^{\varepsilon_{j}} \phi \geq 0$ for all $1 \leq i \leq j \leq n$ and $\varepsilon_{i}, \varepsilon_{j}>0$. Observe that for $1 \leq l \leq j \leq n_{1}$,

$$
\begin{aligned}
& \Delta_{l}^{\varepsilon_{l}} \Delta_{j}^{\varepsilon_{j}} \psi\left(\left(u_{1}^{1}, \ldots, u_{n_{1}}^{1}\right), \ldots,\left(u_{1}^{k}, \ldots, u_{n_{k}}^{k}\right)\right) \\
&=\varphi\left(\sum_{i=1}^{n_{1}} u_{i}^{1}+\varepsilon_{l}+\varepsilon_{j}, \ldots, \sum_{i=1}^{n_{k}} u_{i}^{k}\right)+\varphi\left(\sum_{i=1}^{n_{1}} u_{i}^{1}, \ldots, \sum_{i=1}^{n_{k}} u_{i}^{k}\right) \\
&-\varphi\left(\sum_{i=1}^{n_{1}} u_{i}^{1}+\varepsilon_{l}, \ldots, \sum_{i=1}^{n_{k}} u_{i}^{k}\right)-\varphi\left(\sum_{i=1}^{n_{1}} u_{i}^{1}+\varepsilon_{j}, \ldots, \sum_{i=1}^{n_{k}} u_{i}^{k}\right) \\
& \geq 0
\end{aligned}
$$

from the convexity of $\varphi$ w.r.t. the first coordinate. Similarly for $n_{1}+\ldots+$ $n_{r-1}<l \leq n_{1}+\ldots+n_{r}, n_{1}+\ldots+n_{s-1}<j \leq n_{1}+\ldots+n_{s}$, where $r<s$,

$$
\begin{aligned}
\Delta_{l}^{\varepsilon_{l}} & \Delta_{j}^{\varepsilon_{j}} \psi\left(\left(u_{1}^{1}, \ldots, u_{n_{1}}^{1}\right), \ldots,\left(u_{1}^{k}, \ldots, u_{n_{k}}^{k}\right)\right) \\
= & \varphi\left(\ldots, \sum_{i=1}^{n_{r}} u_{i}^{r}+\varepsilon_{l}, \ldots, \sum_{i=1}^{n_{s}} u_{i}^{s}+\varepsilon_{j}, \ldots\right)+\varphi\left(\ldots, \sum_{i=1}^{n_{r}} u_{i}^{r}, \ldots, \sum_{i=1}^{n_{s}} u_{i}^{s}, \ldots\right) \\
& -\varphi\left(\ldots, \sum_{i=1}^{n_{r}} u_{i}^{r}+\varepsilon_{l}, \ldots, \sum_{i=1}^{n_{s}} u_{i}^{s}, \ldots\right) \\
& -\varphi\left(\ldots, \sum_{i=1}^{n_{r}} u_{i}^{r}, \ldots, \sum_{i=1}^{n_{s}} u_{i}^{s}+\varepsilon_{j}, \ldots\right)
\end{aligned}
$$

$\geq 0$

from the supermodularity of $\varphi$.

3. Main results. We start with some technical lemmas. The first one is a straightforward consequence of the definition of supermodular functions (cf. [1]).

LEMma 3.1. Let $\left\{U_{n}^{i}\right\}_{n \geq 1}, i=1, \ldots, k$, be sequences of nonnegative random variables. Then for all $\varphi \in \mathcal{L}_{\mathrm{sm}}$, the function

$$
\psi\left(n_{1}, \ldots, n_{k}\right)=\mathbb{E}\left[\varphi\left(\sum_{i=1}^{n_{1}} U_{i}^{1}, \ldots, \sum_{i=1}^{n_{k}} U_{i}^{k}\right)\right]
$$

is supermodular on $\mathbb{N}^{k}$.

Lemma 3.2. Let $\left\{U_{n}^{i}\right\}_{n \geq 1}, i=1, \ldots, k$, be stationary sequences of nonnegative random variables such that for all $i \neq j,\left\{U_{n}^{i}\right\}_{n \geq 1}$ is independent of $\left\{U_{n}^{j}\right\}_{n \geq 1}$. Define 


$$
\psi\left(n_{1}, \ldots, n_{k}\right)=\mathbb{E}\left[\varphi\left(\sum_{i=1}^{n_{1}} U_{i}^{1}, \ldots, \sum_{i=1}^{n_{k}} U_{i}^{k}\right)\right] .
$$

(i) If $\varphi \in \mathcal{L}_{\text {dcx (resp. idcx) }}$ then $\psi \in \mathcal{L}_{\text {dcx (resp. idcx) }}$ on $\mathbb{N}^{k}$.

(ii) If $\varphi \in \mathcal{L}_{\text {ccx (resp. iccx) }}$ then $\psi \in \mathcal{L}_{\text {ccx (resp. iccx) }}$ on $\mathbb{N}^{k}$.

(iii) If $\varphi \in \mathcal{L}_{\text {uo-cx }}$ then $\psi \in \mathcal{L}_{\text {uo-cx }}$ on $\mathbb{N}^{k}$.

Proof. (i) Let $\varphi \in \mathcal{L}_{\mathrm{dcx}}$. First, Lemma 3.1 shows that $\psi$ is supermodular. It remains to show that $\psi$ is convex w.r.t. $n_{i}, i=1, \ldots, k$, i.e. it is coordinatewise convex. Let $\mathbf{U}_{n_{i}}^{i}=\left(U_{1}^{i}, \ldots, U_{n_{i}}^{i}\right)$ and write $f^{(n)}\left(u_{1}, \ldots, u_{n}\right)=\sum_{i=1}^{n} u_{i}$. Then, by independence,

$$
\begin{aligned}
& \psi\left(n_{1}+1, n_{2}, \ldots, n_{k}\right)+\psi\left(n_{1}-1, n_{2}, \ldots, n_{k}\right)-2 \psi\left(n_{1}, \ldots, n_{k}\right) \\
&=\mathbb{E}\left[\varphi\left(f^{\left(n_{1}+1\right)}\left(\mathbf{U}_{n_{1}+1}^{1}\right), f^{\left(n_{2}\right)}\left(\mathbf{U}_{n_{2}}^{2}\right), \ldots, f^{\left(n_{k}\right)}\left(\mathbf{U}_{n_{k}}^{k}\right)\right)\right] \\
&+\mathbb{E}\left[\varphi\left(f^{\left(n_{1}-1\right)}\left(\mathbf{U}_{n_{1}-1}^{1}\right), f^{\left(n_{2}\right)}\left(\mathbf{U}_{n_{2}}^{2}\right), \ldots, f^{\left(n_{k}\right)}\left(\mathbf{U}_{n_{k}}^{k}\right)\right)\right] \\
&-2 \mathbb{E}\left[\varphi\left(f^{\left(n_{1}\right)}\left(\mathbf{U}_{n_{1}}^{1}\right), f^{\left(n_{2}\right)}\left(\mathbf{U}_{n_{2}}^{2}\right), \ldots, f^{\left(n_{k}\right)}\left(\mathbf{U}_{n_{k}}^{k}\right)\right)\right] \\
&= \mathbb{E}\left[\varphi\left(f^{\left(n_{1}+1\right)}\left(\mathbf{U}_{n_{1}+1}^{1}\right), f^{\left(n_{2}\right)}\left(\mathbf{U}_{n_{2}}^{2}\right), \ldots, f^{\left(n_{k}\right)}\left(\mathbf{U}_{n_{k}}^{k}\right)\right)\right] \\
&+\mathbb{E}\left[\varphi\left(f^{\left(n_{1}+1\right)}\left(0, U_{1}^{1}, \ldots, U_{n_{1}-1}^{1}, 0\right), f^{\left(n_{2}\right)}\left(\mathbf{U}_{n_{2}}^{2}\right), \ldots, f^{\left(n_{k}\right)}\left(\mathbf{U}_{n_{k}}^{k}\right)\right)\right] \\
&-\mathbb{E}\left[\varphi\left(f^{\left(n_{1}+1\right)}\left(0, U_{1}^{1}, \ldots, U_{n_{1}}^{1}\right), f^{\left(n_{2}\right)}\left(\mathbf{U}_{n_{2}}^{2}\right), \ldots, f^{\left(n_{k}\right)}\left(\mathbf{U}_{n_{k}}^{k}\right)\right)\right] \\
&-\mathbb{E}\left[\varphi\left(f^{\left(n_{1}+1\right)}\left(U_{1}^{1}, \ldots, U_{n_{1}}^{1}, 0\right), f^{\left(n_{2}\right)}\left(\mathbf{U}_{n_{2}}^{2}\right), \ldots, f^{\left(n_{k}\right)}\left(\mathbf{U}_{n_{k}}^{k}\right)\right)\right] \\
&=\mathbb{E} {\left[\varphi\left(f^{\left(n_{1}+1\right)}\left(\mathbf{U}_{n_{1}+1}^{1}\right), f^{\left(n_{2}\right)}\left(\mathbf{U}_{n_{2}}^{2}\right), \ldots, f^{\left(n_{k}\right)}\left(\mathbf{U}_{n_{k}}^{k}\right)\right)\right] } \\
&+\mathbb{E}\left[\varphi\left(f^{\left(n_{1}+1\right)}\left(0, U_{2}^{1}, \ldots, U_{n_{1}}^{1}, 0\right), f^{\left(n_{2}\right)}\left(\mathbf{U}_{n_{2}}^{2}\right), \ldots, f^{\left(n_{k}\right)}\left(\mathbf{U}_{n_{k}}^{k}\right)\right)\right] \\
&-\mathbb{E}\left[\varphi\left(f^{\left(n_{1}+1\right)}\left(0, U_{2}^{1}, \ldots, U_{n_{1}+1}^{1}\right), f^{\left(n_{2}\right)}\left(\mathbf{U}_{n_{2}}^{2}\right), \ldots, f^{\left(n_{k}\right)}\left(\mathbf{U}_{n_{k}}^{k}\right)\right)\right] \\
&-\mathbb{E}\left[\varphi\left(f^{\left(n_{1}+1\right)}\left(U_{1}^{1}, \ldots, U_{n_{1}}^{1}, 0\right), f_{2}^{\left(n_{2}\right)}\left(\mathbf{U}_{n_{2}}^{2}\right), \ldots, f_{k}^{\left(n_{k}\right)}\left(\mathbf{U}_{n_{k}}^{k}\right)\right)\right] .
\end{aligned}
$$

In the second equality we used the symmetry of $f^{(n)}$, whereas in the third we used stationarity. Write the above equation in the form

$$
\begin{aligned}
& \psi\left(n_{1}+1, n_{2}, \ldots, n_{k}\right)+\psi\left(n_{1}-1, n_{2}, \ldots, n_{k}\right)-2 \psi\left(n_{1}, \ldots, n_{k}\right) \\
& =\int \mathbb{E}\left[\varphi\left(f^{\left(n_{1}+1\right)}\left(\mathbf{U}_{n_{1}+1}^{1}\right), f^{\left(n_{2}\right)}\left(\mathbf{u}_{n_{2}}^{2}\right), \ldots, f^{\left(n_{k}\right)}\left(\mathbf{u}_{n_{k}}^{k}\right)\right)\right] d \mathbb{P}^{\mathbf{U}^{1}}\left(\mathbf{u}^{1}\right) \\
& \quad+\int \mathbb{E}\left[\varphi\left(f^{\left(n_{1}+1\right)}\left(0, U_{2}^{1}, \ldots, U_{n_{1}}^{1}, 0\right), f^{\left(n_{2}\right)}\left(\mathbf{u}_{n_{2}}^{2}\right), \ldots, f^{\left(n_{k}\right)}\left(\mathbf{u}_{n_{k}}^{k}\right)\right)\right] d \mathbb{P}^{\mathbf{U}^{1}}\left(\mathbf{u}^{1}\right) \\
& \quad-\int \mathbb{E}\left[\varphi\left(f^{\left(n_{1}+1\right)}\left(0, U_{2}^{1}, \ldots, U_{n_{1}+1}^{1}\right), f^{\left(n_{2}\right)}\left(\mathbf{u}_{n_{2}}^{2}\right), \ldots, f^{\left(n_{k}\right)}\left(\mathbf{u}_{n_{k}}^{k}\right)\right)\right] d \mathbb{P}^{\mathbf{U}^{1}}\left(\mathbf{u}^{1}\right) \\
& \quad-\int \mathbb{E}\left[\varphi\left(f^{\left(n_{1}+1\right)}\left(U_{1}^{1}, \ldots, U_{n_{1}}^{1}, 0\right), f^{\left(n_{2}\right)}\left(\mathbf{u}_{n_{2}}^{2}\right), \ldots, f^{\left(n_{k}\right)}\left(\mathbf{u}_{n_{k}}^{k}\right)\right)\right] d \mathbb{P}^{\mathbf{U}^{1}}\left(\mathbf{u}^{1}\right) .
\end{aligned}
$$


Here $\mathbb{P}^{\mathbf{U}^{1}}$ denotes the distribution of $\left(\mathbf{U}_{n_{2}}^{2}, \ldots, \mathbf{U}_{n_{k}}^{k}\right)$ and $\mathbf{u}^{1} \equiv\left(\mathbf{u}_{n_{2}}^{2}, \ldots, \mathbf{u}_{n_{k}}^{k}\right)$. Because for all $n \geq 1, f^{(n)}$ is linear and $\varphi$ is convex w.r.t. the first coordinate (because it is directionally convex) we deduce that

$$
\varphi\left(f^{\left(n_{1}+1\right)}\left(u_{1}^{1}, \ldots, u_{n_{1}}^{1}, u_{n_{1}+1}^{1}\right), f^{\left(n_{2}\right)}\left(\mathbf{u}_{n_{2}}^{2}\right), \ldots, f^{\left(n_{k}\right)}\left(\mathbf{u}_{n_{k}}^{k}\right)\right)
$$

is supermodular w.r.t. $\left(u_{1}^{1}, \ldots, u_{n_{1}+1}^{1}\right)$ (Lemma 2.2(i)). Therefore

$$
\psi\left(n_{1}+1, n_{2}, \ldots, n_{k}\right)+\psi\left(n_{1}-1, n_{2}, \ldots, n_{k}\right)-2 \psi\left(n_{1}, \ldots, n_{k}\right) \geq 0,
$$

which ends the proof.

(ii) The proof is similar to (i).

(iii) Let $\varphi \in \mathcal{L}_{\text {uo-cx }}$. Then $\psi\left(n_{1}, \ldots, n_{k}\right)$ can be written in the form

$$
\psi\left(n_{1}, \ldots, n_{k}\right)=\mathbb{E}\left[\prod_{j=1}^{k} h_{j}\left(\sum_{i=1}^{n_{j}} U_{i}^{j}\right)\right],
$$

where the $h_{j}$ are increasing and convex. From independence we have

$$
\psi\left(n_{1}, \ldots, n_{k}\right)=\prod_{j=1}^{k} \mathbb{E}\left[h_{j}\left(\sum_{i=1}^{n_{j}} U_{i}^{j}\right)\right] .
$$

Now, by (i) with $k=1$, the functions $g_{j}\left(n_{j}\right)=\mathbb{E}\left[h_{j}\left(\sum_{i=1}^{n_{j}} U_{i}^{j}\right)\right], j=1, \ldots, k$, are increasing and convex. Therefore $\psi\left(n_{1}, \ldots, n_{k}\right)$ can be written as a product of increasing and convex functions, which ends the proof.

In all cases the monotonicity is obvious.

Using the similar argument (with Lemma 2.2(ii)) we can prove the following lemma.

Lemma 3.3. Let $\left\{U_{n}^{i}\right\}_{n \geq 1}, i=1, \ldots, k$, be jointly stationary sequences

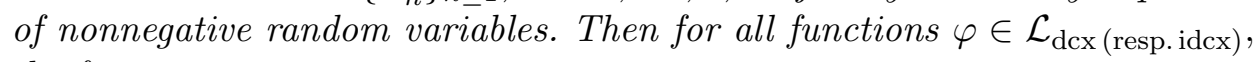
the function

$$
\psi(n)=\mathbb{E}\left[\varphi\left(\sum_{i=1}^{n} U_{i}^{1}, \ldots, \sum_{i=1}^{n} U_{i}^{k}\right)\right]
$$

is convex (resp. increasing and convex) on $\mathbb{N}$.

REMARK 3.4. All the above results in the case $k=1$ mean that $\psi(n)=$ $\varphi\left(\sum_{i=1}^{n} U_{i}\right)$ is convex for convex $\varphi$. This result was proved in $[9$, p. 278] for $\left\{U_{n}\right\}_{n \geq 1}$ being a sequence of iid nonnegative random variables. In [2] it was shown for nonstationary sequences of independent nonnegative random variables such that $\mathbb{E}\left[h\left(U_{n}\right)\right] \leq \mathbb{E}\left[h\left(U_{n+1}\right)\right]$ for all $h \in \mathcal{L}_{\text {cx (resp. icx). Makowski }}$ and Phillips [5] showed that for iid nonnegative random variables $\left\{U_{n}\right\}_{n \geq 1}$, the function $\psi(n) / n$ is increasing.

From the above lemmas we have directly the following theorems. Let

$$
\mathbf{N}=\left(N_{1}, \ldots, N_{k}\right), \quad \widetilde{\mathbf{N}}=\left(\widetilde{N}_{1}, \ldots, \widetilde{N}_{k}\right)
$$


be vectors of nonnegative, integer-valued random variables and

$$
\mathbf{T}=\left(\sum_{i=1}^{N_{1}} U_{i}^{1}, \ldots, \sum_{i=1}^{N_{k}} U_{i}^{k}\right), \quad \widetilde{\mathbf{T}}=\left(\sum_{i=1}^{\widetilde{N}_{1}} U_{i}^{1}, \ldots, \sum_{i=1}^{\widetilde{N}_{k}} U_{i}^{k}\right) .
$$

THEOREM 3.5. Under the assumptions of Lemma 3.2:

(i) If $\mathbf{N}<_{\operatorname{dcx}(\text { resp. idcx) }} \widetilde{\mathbf{N}}$ then $\mathbf{T}<_{\operatorname{dcx}(\text { resp. idcx) }} \widetilde{\mathbf{T}}$.

(ii) If $\mathbf{N}<_{\text {ccx (resp. iccx) }} \tilde{\mathbf{N}}$ then $\mathbf{T}<_{\text {ccx (resp. iccx) }} \widetilde{\mathbf{T}}$.

(iii) If $\mathbf{N}<_{\text {uo-cx }} \tilde{\mathbf{N}}$ then $\mathbf{T}<_{\text {uo-cx }} \widetilde{\mathbf{T}}$.

Let now $N, \tilde{N}$ be nonnegative and integer-valued random variables.

THEOREM 3.6. Under the assumptions of Lemma 3.3, if $N<_{\mathrm{cx} \text { (resp. icx) }}$ $\widetilde{N}$ then

$$
\left(\sum_{i=1}^{N} U_{i}^{1}, \ldots, \sum_{i=1}^{N} U_{i}^{k}\right)<_{\mathrm{dcx}(\operatorname{resp} . \mathrm{idcx})}\left(\sum_{i=1}^{\tilde{N}} U_{i}^{1}, \ldots, \sum_{i=1}^{\tilde{N}} U_{i}^{k}\right) .
$$

Pellerey [8] showed the following preservation results which are parallel to Theorem 3.5. Assume that $\left\{U_{n}^{i}\right\}_{n \geq 1}, i=1, \ldots, k$, are independent sequences of nonnegative independent random variables such that for each $f \in \mathcal{L}_{\text {cx (resp. icx) }}, \mathbb{E}\left[f\left(U_{n}^{i}\right)\right] \leq \mathbb{E}\left[f\left(U_{n+1}^{i}\right)\right], n \geq 1, i=1, \ldots, k$. Then $\mathbf{N}<_{\text {ccx }} \tilde{\mathbf{N}}$ implies $\mathbf{T}<_{\text {ccx }} \widetilde{\mathbf{T}}\left(\mathbf{N}<_{\text {icx (resp. iccx, uo-cx })} \widetilde{\mathbf{N}}\right.$ implies $\left.\mathbf{T}<_{\text {icx }(\text { resp. iccx, uo-cx })} \widetilde{\mathbf{T}}\right)$. Moreover, without any independence assumption, $\mathbf{N}<_{\text {uo (resp. lo) }} \widetilde{\mathbf{N}}$ implies $\mathbf{T}<_{\text {uo (resp.lo) }} \widetilde{\mathbf{T}}$. In [1] it was shown under the same assumptions that $\mathbf{N}<_{\text {sm }} \widetilde{\mathbf{N}}$ implies $\mathbf{T}<_{\text {sm }} \widetilde{\mathbf{T}}$.

4. Criteria for variability orderings. In this section we point out some situations when the sufficient conditions of Theorem 3.5 are satisfied, i.e. we present examples of integer-valued random variables $N_{1}, \ldots, N_{k}$, $\widetilde{N}_{1}, \ldots, \widetilde{N}_{k}$ such that $\mathbf{N}<_{a} \widetilde{\mathbf{N}}$, where $<_{\text {a }}$ is one of the above orderings.

(i) Assume that for integer-valued random variables we have $N<_{\text {icx }} \widetilde{N}$. For example we can take $N:=N(t), t \geq 0$, where $(N(t), t \geq 0)$ is a stationary counting process $N(t):=\sum_{n=0}^{\infty} \mathbb{I}\left(T_{n} \leq t\right)$ for which the interpoint distances $X_{n}=T_{n}-T_{n-1}, n \geq 1$, have distribution $F_{X}$ and the first distance $T_{0}$ from the origin has distribution

$$
F_{X}^{r}(x):=\frac{1}{E\left[X_{1}\right]} \int_{0}^{x}\left(1-F_{X}(u)\right) d u
$$

In a similar way we define elements with tildes. If

$$
\sum_{i=1}^{n} X_{i}<_{\mathrm{cx}} \sum_{i=1}^{n} \widetilde{X}_{i} \quad \text { for all } n \geq 1
$$


then $N(t)<_{\text {cx }} \tilde{N}(t)$ for all $t \geq 0$ (cf. [3]). For instance, (1) holds if

$$
\left(X_{1}, \ldots, X_{n}\right)<_{\operatorname{dcx}}\left(\widetilde{X}_{1}, \ldots, \widetilde{X}_{n}\right) \quad \text { for all } n \geq 1,
$$

i.e. the sequence of interpoint distances in the second counting process is more dependent and more variable than in the first one. We refer to [3] for examples. In the case of renewal counting processes we can take as $X_{n}$ and $\widetilde{X}_{n}$ any positive random variables which are ordered w.r.t. $<_{\mathrm{cx}}$ (note that $<_{\mathrm{cx}}$ is closed under convolution).

Another possibility to obtain $N<_{\text {cx }} \widetilde{N}$ is the following. Assume that $N$ and $\widetilde{N}$ have finite support. Then $N<_{\text {cx }} \widetilde{N}$ if and only if the probability function of $\widetilde{N}$ can be obtained from $N$ by a finite sequence of local mean preserving spreads, which means, roughly speaking, that the mass is removed from a point $x$ in the support of $N$ and shifted to $y$ and $z(y<x<z)$ in the support of $\widetilde{N}$, but the mean remains constant (see [7, Definition 1.5.28 and Theorem 1.5.29] for more details).

Let now $\left\{V_{n}\right\}_{n \geq 1}$ be a stationary sequence of $\{1, \ldots, k\}$-valued random variables. For $j=1, \ldots, k$ define

$$
N_{j}=\sum_{i=1}^{N} \mathbb{I}\left(V_{i}=j\right), \quad \widetilde{N}_{j}=\sum_{i=1}^{\widetilde{N}} \mathbb{I}\left(V_{i}=j\right) .
$$

Then using Theorem 3.6 with $U_{i}^{j}=\mathbb{I}\left(V_{i}=j\right)$ we have $\mathbf{N}<_{\text {dcx }} \tilde{\mathbf{N}}$.

(ii) Consider a vector $\mathbf{N}=\left(N_{1}, \ldots, N_{k}\right)$ of integer-valued random variables with the same marginal distribution $F$. It follows from the Lorentz inequality $([11])$ that $\left(N_{1}, \ldots, N_{k}\right)<_{\mathrm{dcx}}\left(N_{1}, \ldots, N_{1}\right)$.

(iii) Assume that $N_{i}<_{\mathrm{cx}} \widetilde{N}_{i}, i=1, \ldots, k$. If $\mathbf{N}$ and $\widetilde{\mathbf{N}}$ are comonotone random vectors or have a common conditionally increasing copula then $\mathbf{N}<_{\text {dcx }} \widetilde{\mathbf{N}}$ (see [7, Lemma 3.12.13 and Theorem 3.12.14] for more details).

\section{References}

[1] M. Denuit, C. Genest and É. Marceau, Criteria for the stochastic ordering of random sums, with actuarial applications, Scand. Actuar. J. 2002, 3-16.

[2] A. Jean-Marie and Z. Liu, Stochastic comparison for queueing models via random sums and intervals, Adv. Appl. Probab. 24 (1992), 960-985.

[3] R. Kulik and R. Szekli, Sufficient conditions for long-range dependence of stationary point processes on the real line, J. Appl. Probab. 38 (2001), 570-581.

[4] - - - Dependence orderings for some functionals of multivariate point processes, J. Multivariate Anal. (2004), to appear.

[5] A. Makowski and T. Philips, Stochastic convexity of sums of i.i.d. non-negative random variables with applications, J. Appl. Probab. 29 (1992), 156-167.

[6] A. W. Marshall and I. Olkin, Inequalities: Theory of Majorization and Its Applications, Academic Press, 1979. 
[7] A. Müller and D. Stoyan, Comparison Methods for Stochastic Models and Risks, Wiley, New York, 2002.

[8] F. Pellerey, Stochastic comparisons for multivariate shock models, J. Multivariate Anal. 71 (1999), 42-55.

[9] S. Ross, Stochastic Processes, Wiley, New York, 1986.

[10] M. Shaked and J.G. Shanthikumar, Stochastic Orders and Their Applications, Academic Press, London, 1994.

[11] - - - Supermodular stochastic orders and positive dependence of random vectors, J. Multivariate Anal. 61 (1997), 86-101.

[12] T. Wong, Preservation of multivariate stochastic orders under multivariate Poisson shock models, J. Appl. Probab. 34 (1997), 1009-1020.

Mathematical Institute

Wrocław University

Pl. Grunwaldzki 2/4

50-384 Wrocław, Poland

E-mail: rkuli@math.uni.wroc.pl

Received on 19.6.2002;

revised version on 4.3.2003 